\title{
Antiphospholipid antibodies, systemic lupus erythematosus, and non-traumatic metatarsal fractures
}

\author{
S Sangle, D P D'Cruz, M A Khamashta, G R V Hughes
}

Ann Rheum Dis 2004;63:1241-1243. doi: 10.1136/ard.2003.016105

See end of article for authors' affiliations

Correspondence to:

Dr D P D'Cruz, The Lupus

Research Unit, The Rayne

Institute, St Thomas'

Hospital, London SEI 7EH,

UK; david.d'cruz@

kcl.ac.uk

Accepted 1

December 2003
Background: Stress fractures are common in military recruits and athletes and are thought to be secondary to stress and overuse. Less often they are associated with metabolic disorders such as osteoporosis, hypophosphataemia, and diabetes mellitus.

Objective: Analysis of 19 patients with systemic lupus erythematosus and antiphospholipid antibodies presenting consecutively with foot pain. All had metatarsal fractures (six bilateral) without any obvious history of trauma.

Results: 13 of the 19 patients had antiphospholipid syndrome. Among the whole group, 13 had normal bone mineral density, one had osteopenia, and five others had osteoporosis as defined by WHO criteria; 10 had received steroids, mostly in low dosage; 13 were receiving warfarin. There was no evidence that a metabolic bone abnormality was a unifying factor in the pathogenesis.

Conclusions: Atraumatic metatarsal stress fractures may occur in SLE, particularly in association with the antiphospholipid syndrome. The pathogenesis of these fractures remains uncertain but microinfarcts in the metatarsal bones are a possible cause.
S tress fractures of the metatarsals are thought to be caused by overuse injury, but their aetiology is not well understood. ${ }^{1}$ It is postulated that high bone strain and repetitive submaximal stress leads to microfractures. Stress fractures occur more often in women, and additional intrinsic factors such as hormonal imbalance may also contribute. ${ }^{2}$ Metatarsal fractures are described in metabolic disorders such as osteoporosis, hypophosphataemia, and diabetes mellitus. To our knowledge metatarsal fractures have not previously been reported in patients with systemic lupus erythematosus (SLE) and antiphospholipid syndrome. We describe the clinical and radiological features of metatarsal fractures in patients with SLE associated with antiphospholipid syndrome and in patients with antiphospholipid antibodies (aPL) alone.

\section{METHODS}

We describe 19 patients with SLE with or without antiphospholipid syndrome, who subsequently developed metatarsal fractures. All were regular outpatients at the Louise Coote Lupus Unit at St Thomas' Hospital. The patients were seen over the previous year and represent consecutive patients presenting to the clinic with foot pain. Our cohort of 2500 patients with connective tissue disease was not specifically screened for patients with foot pain.

All patients were female, with a mean age of 42 years (range 24 to 58). Seventeen had systemic lupus erythematosus (SLE) as classified by the ACR criteria. ${ }^{3}$ Thirteen of these 17 SLE patients had antiphospholipid syndrome and two were positive for aPL. Two had primary antiphospholipid syndrome. Thus 17 of 19 patients had positive aPL and 15 of these patients fulfilled the Sapporo classification criteria for antiphospholipid syndrome. ${ }^{4}$ Thirteen of these patients with antiphospholipid syndrome were anticoagulated with warfarin, with a target INR of 3.0-4.0. Five patients had previously received prednisolone $(>15 \mathrm{mg}$ ) for more than six months. Only one had a history of a trivial foot injury associated with the fracture. One patient had type I diabetes in addition to SLE and antiphospholipid syndrome. All the patients were assessed for the duration of their autoimmune disease, steroid dose, menopausal status, bone mineral density, chronic metabolic diseases, haemoglobinopathies, and myeloma. The location and characteristics of the metatarsal fractures were determined by radiology and in some cases by radionuclide bone scan.

\section{RESULTS}

Of the 19 patients with metatarsal fractures, six had bilateral fractures (figs 1 and 2). One patient had diabetes mellitus, but her metabolic bone profile and bone mineral density were normal. Five patients had osteoporosis and one had osteopenia, while the other 13 had normal bone mineral density (table 1). One patient with primary antiphospholipid syndrome had avascular necrosis of the second and third metatarsal heads. A further patient with SLE and antiphospholipid syndrome on prednisolone had bilateral avascular necrosis of the femoral head as well as metatarsal fractures. One of the two patients with primary antiphospholipid syndrome, who had never received steroids, had bilateral metatarsal fractures. Apart from the one patient with type I diabetes mellitus, none had any chronic metabolic disease, malignancies, or haemoglobinopathies. All patients had normal serum calcium, alkaline phosphate, and phosphate levels.

\section{DISCUSSION}

Metatarsal fractures (fatigue fractures) are common in military personnel. They are also seen in athletes, but much less so in civilian practice. Metatarsal fractures are described in metabolic disorders such as osteoporosis, hypophosphataemia, osteoporosis, and diabetes mellitus. There is a correlation between metatarsal fractures and low bone mineral density in both sexes, confirmed by the low DEXA scan findings. ${ }^{5}$ Recently, metatarsal fracture has been reported in patients with rheumatoid arthritis and chronic bronchitis. ${ }^{6}$ Elkayam et al described eight patients with

Abbreviations: aPL, antiphospholipid antibodies; DEXA, dual energy $x$ ray absorptiometry; INR, international normalised ratio; SLE, systemic lupus erythematosus 


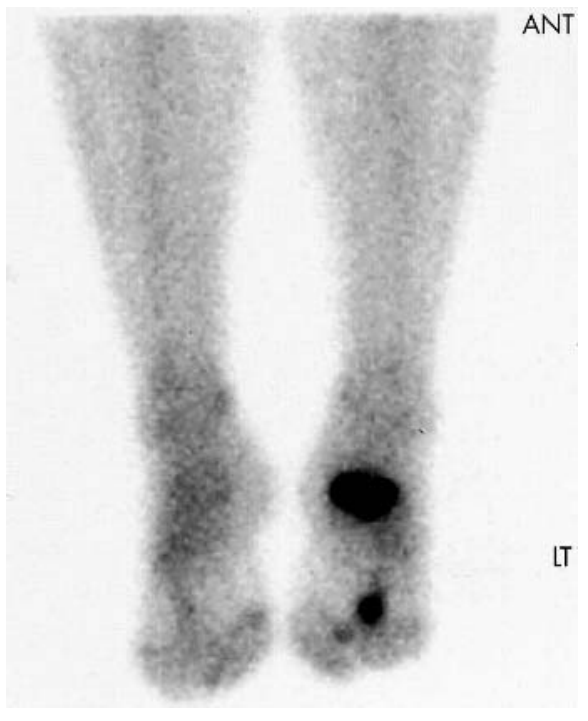

Figure 1 A radionuclide scan of the foot showing an area of increased uptake suggesting metatarsal fracture in a patient with primary antiphospholipid syndrome.

rheumatoid arthritis and one with polymyalgia rheumatica who developed stress insufficiency fractures. All were treated with corticosteroids and had low bone mineral density. ${ }^{7}$

Here we describe 19 patients with SLE who had metatarsal fractures, six of which were bilateral. Only one had any history of trivial injury five years previously. Interestingly, 13 of our patients had normal bone mineral density values on DEXA scanning, one had osteopenia, and five others had osteoporosis as defined by World Health Organisation criteria. This finding goes against the hypothesis that osteoporosis was the sole cause of metatarsal fractures in these patients. Furthermore, six of the patients had bilateral metatarsal fractures without any obvious history of preceding trauma, and only one of these had osteoporosis, arguing against the concept of repeated trauma leading to metatarsal fractures, as seen in military personnel and sports injuries.

One patient had diabetes mellitus type I with secondary antiphospholipid syndrome and was well controlled on insulin; her DEXA scan showed normal bone mineral density. Her metabolic profile was normal. Clearly other factors may have been important in explaining the metatarsal fractures in this patient.

All patients were screened for metabolic disorders, endocrine disorders, and malignancies including multiple myeloma. Five of the patients had osteoporosis and one had

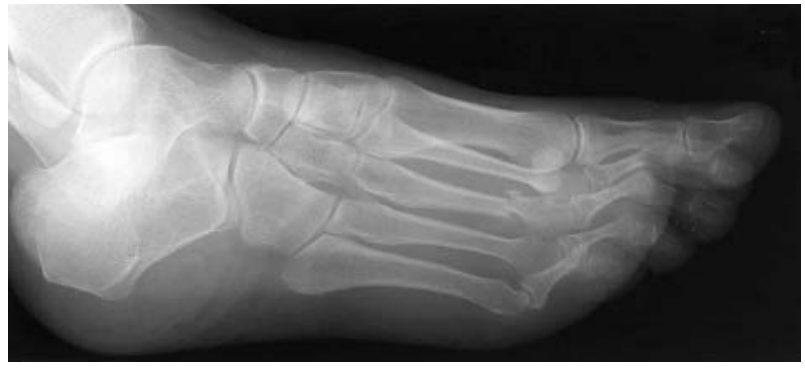

Figure 2 Foot $x$ ray showing metatarsal fracture in a patient with secondary antiphospholipid syndrome.

diabetes mellitus, while one had SLE nephritis with antiphospholipid syndrome and was on high doses of steroids $(>15$ mg/day). This latter patient had avascular necrosis of the hips, and her bone mineral density was in the osteoporotic range. It is possible that in this patient three or more factors were responsible for the metatarsal fractures. However, the other 14 patients (including the one with diabetes mellitus) did not have any metabolic bone abnormality. This further reduces the possibility that metabolic disorders alone were the main cause of metatarsal fractures in these patients.

Thirteen patients with antiphospholipid syndrome were anticoagulated with warfarin, with a target INR of 3.0 to 4.0. This raises the possibility that warfarin may have played a role in the fractures. Two recent papers have studied the association between long term warfarin, a vitamin $\mathrm{K}$ antagonist, and osteoporosis. ${ }^{89}$ The results were contradictory, one showing an association between warfarin and osteoporosis while the other (involving 6000 postmenopausal women on warfarin) found no increased risk of osteoporosis. In our cohort, two patients with secondary antiphospholipid syndrome were on long term warfarin (for more than one year) and had received steroids (more than $15 \mathrm{mg} /$ day). Both had osteoporosis. It therefore remains unclear whether warfarin played a role in the development of metatarsal fractures in our patients.

An increased frequency of non-traumatic metatarsal fractures in the patients with SLE has not been reported before. In general, it would be easy to blame osteoporosis caused by the disease or by steroid treatment, and indeed five of our patients showed evidence of osteoporosis. However, other factors are certainly important in the pathogenesis of these fractures. Thus, while 10 patients had received steroids in their disease course, eight had been on low doses $(<7.5 \mathrm{mg} /$ day $)$, which makes it less likely that this was the major cause of the metatarsal fractures. None of these patients had experienced undue physical stress or repetitive or overuse injuries.

Table 1 Metatarsal fractures in patients with systemic lupus erythematosus and antiphospholipid syndrome

\begin{tabular}{|c|c|c|c|c|c|c|c|c|c|}
\hline Patients & $\begin{array}{l}\text { Unilateral } \\
\text { fractures }\end{array}$ & $\begin{array}{l}\text { Bilateral } \\
\text { fractures }\end{array}$ & $\begin{array}{l}\text { Osteoporosis } \\
\text { (mean T and } \\
\text { Z scores) }\end{array}$ & $\begin{array}{l}\text { Normal } \\
\text { BMD (no } \\
\text { osteoporosis, } \\
n=14 \text { ) }\end{array}$ & $\begin{array}{l}\text { Chronic } \\
\text { metabolic } \\
\text { disease }\end{array}$ & $\begin{array}{l}\text { Serum } \\
\text { calcium*; } \\
\text { normal range, } \\
2.20- \\
2.60 \mathrm{mmol} / \mathrm{l}\end{array}$ & $\begin{array}{l}\text { Serum } \\
\text { alkaline } \\
\text { phosphatase; } \\
\text { normal } \\
\text { range, } \\
31-116 \mathrm{IU} / \mathrm{I}\end{array}$ & $\begin{array}{l}\text { Serum } \\
\text { phosphate; } \\
\text { normal range, } \\
0.8- \\
1.15 \mathrm{mmol} / \mathrm{I}\end{array}$ & $\begin{array}{l}\text { Prednisolone } \\
>15 \mathrm{mg} / \mathrm{d}\end{array}$ \\
\hline Primary APS $(n=2)$ & 1 & 1 & & 2 & $\mathrm{Nil}$ & 2.26 & 84 & 1.3 & None \\
\hline SLE and $a P L+(n=15)$ & 11 & 4 & $\begin{array}{l}4 \\
T=-2.7 \\
Z=-2.65\end{array}$ & 11 & 1 (DM) & 2.34 & 78 & 1.4 & 4 \\
\hline SLE and $a P L-(n=2)$ & 1 & 1 & $\begin{array}{l}\mathrm{T}=-2.85 ; \\
\mathrm{Z}=-2.72\end{array}$ & 1 & Nil. & 2.35 & 113 & 1.1 & 1 \\
\hline
\end{tabular}

*Corrected for albumin.

APS, antiphospholipid syndrome; BMD, bone mineral density; DM, diabetes mellitus; SLE, systemic lupus erythematosus. 
The precise pathogenesis of metatarsal fractures in our patients with SLE and antiphospholipid syndrome remains unclear. The possible contributory factors include submaximal stress or vasculopathy of the vessels supplying the bones, and osteoporosis. Antiphospholipid syndrome (Hughes syndrome) is characterised by arterial-venous thrombosis. ${ }^{10}$ Strikingly 13 of our 19 patients had antiphospholipid antibodies associated with thrombosis. Four patients had raised anticardiolipin antibodies ( $\operatorname{IgG}$ ) and others were positive for lupus anticoagulants. Two aPL positive patients did not fulfil the Sapporo criteria for antiphospholipid syndrome. It is therefore possible that bone microinfarcts may lead to bone damage and to long bone fractures in the feet.

Avascular necrosis of the hips is well described in patients with sickle cell disease and results from bone infarction in the femoral head. Asherson et al reported that avascular necrosis of the hip was twice as common in lupus patients with antiphospholipid syndrome as in those without antiphospholipid syndrome. ${ }^{11}$ However, Houssiau et al failed to show an increased prevalence of avascular necrosis in patients with aPL. ${ }^{12}$ Recently, Tektonidou et al found asymptomatic avascular necrosis of femoral heads in almost $20 \%$ of patients with antiphospholipid syndrome. ${ }^{13}$ Asherson described osteonecrosis of bone as an unusual manifestation of antiphospholipid syndrome. ${ }^{14}$ Another study showed a correlation between anticardiolipin antibodies and recurrent venous thrombosis, thrombocytopenia, haemolytic anaemia, recurrent fetal loss, cutaneous ulcers, transverse myelitis, and pulmonary hypertension, but no correlation between anticardiolipin antibodies and migraine, transient ischaemic attacks, psychosis, or avascular necrosis of bone. ${ }^{15}$ In our series, only four patients had raised anticardiolipin antibodies in significant titre and the rest were positive for lupus anticoagulant. Recently, Jones et al described the high incidence of osteonecrosis in patients with coagulopathies including anticardiolipin antibodies. ${ }^{16}$ These intriguing findings give further support to the view that microinfarcts in the metatarsal bones could result in fractures in patients with antiphospholipid syndrome and antiphospholipid antibodies, who may already have experienced previous thrombotic events. There was a strong correlation between antiphospholipid antibodies and the metatarsal fractures in our series. However, the role of anticoagulation with warfarin in the pathogenesis of these fractures remains unclear.

Our unit is a referral centre for both SLE and antiphospholipid syndrome and it is possible that referral bias may have influenced our observations. Furthermore this was a case series of consecutive patients attending our clinics with foot pain who on investigation were found to have metatarsal fractures. To ascertain the true prevalence of these fractures and their relation to aPL, a prospective study is clearly needed, screening both symptomatic and asymptomatic patients with SLE and aPL positivity, and comparing them with aPL negative patients.

\section{Authors' affiliations}

S Sangle, D P D'Cruz, M A Khamashta, G R V Hughes, The Lupus Research Unit, The Rayne Institute, Lambeth Wing, St Thomas' Hospital, London, UK

\section{REFERENCES}

1 Donahue SW, Sharky NA. Strains in the metatarsals during the stance phase gait implication for stress fractures. J Bone Joint Surg Am 1999:81:1236-44.

2 Boden BP, Osbahr DC. High risk stress fractures: evaluation and treatment. J Am Acad Orthop Surg 2000;8:344-53.

3 Hochberg MC. Updating the American College of Rheumatology revised criteria for the classification of systemic lupus erythematosus. Arthritis Rheum 1997; 40: 1725.

4 Wilson WA, Haravi AE, Koike T, Lockshin MD, Branch DW, Piette JC, et al. International consensus statement on preliminary classification criteria for definite antiphospholipid syndrome: report of an international workshop. Arthritis Rheum 1999:42:1309-11.

5 Tomzak RL, VanCourt R. Metatarsal insufficiency fractures in previously undiagnosed osteoporosis patients. J Foot Ankle Surg 2000;39:174-83.

6 Ivanosvski A. Medjedovic D, Perisic V. Fracture of metatarsal bones caused by fatigue. Srp Art Celok Lek 1998;126:107-10.

7 Elkayam O, Paran D, Flusser G, Wigler I, Yaron M, Caspi D. Insufficiency fractures in rheumatic patients: misdiagnosis an underlying characteristics. Clin Exp Rheumatol 2000;18:369-74.

8 Philip WJ, Martin JC, Richardson JM, Reid DM, Webster J, Douglas AS. Decreased axial and peripheral bone density in patients taking long-term warfarin. Q J Med 1995;88:635-40.

9 Jamal SA, Browner WS, Bauer DC, Cummings SR. Warfarin use and risk for osteoporosis in elderly women. Ann Intern Med 1998;128:829-32.

10 Hughes GRV. Off the beaten track: a clinician's view. In: Khamashta MA, ed. Hughes syndrome (antiphospholipid syndrome). London: Springer, 2000:105-10.

11 Asherson RA, Liote F, Page B, Meyer O, Buchanan N, Khamashta MA, et al. Avascular necrosis of bone and antiphospholipid antibodies in systemic lupus erythematosus. J Rheumatol 1993;20:284-8.

12 Houssiau FA, N'Zeusseu Toukap A, Depresseux G, Maldague BE, Malghem J, Devogelaer JP, et al. Magnetic resonance imaging-detected avascular osteonecrosis in systemic lupus erythematosus: lack of correlation with antiphospholipid antibodies. Br J Rheumatol 1998;37:448-53.

13 Tektonidou MG, Malagari K, Panayiotis G, Kelekis DA, Moutsopoulos HM. Asymptomatic avascular necrosis in patients with primary antiphospholipid syndrome in the absence of corticosteroid use: a prospective study by magnetic resonance imaging. Arthritis Rheum 2003;48:732-6.

14 Asherson Ra, Cervera R. Unusual manifestations of the antiphospholipid syndrome. Clin Rev Allergy Immunol 2003;25:61-78.

15 Alarcon-Segovia D, Deleze M, Oria CV, Sanchez-Guerrero J, GomezPacheco L, Cabiedes J, et al. Antiphospholipid antibodies and the antiphospholipid syndrome in systemic lupus erythematosus. A prospective analysis of 500 consecutive patients. Medicine 1989;68:353-65.

16 Jones LC, Mont MA, Le TB, Petri M, Hungerford DS, Wang P, et al. Procoagulants and osteonecrosis. J Rheumatol 2003;30:783. 